\title{
Effect of interatomic potentials on mass transfer by supersonic 2-crowdions
}

\author{
E. A. Korznikova ${ }^{1}$, I. R. Sunagatova ${ }^{2}$, A. M. Bayazitov ${ }^{2}$, A. S. Semenov ${ }^{3}$, S. V. Dmitriev ${ }^{\dagger, 1,4}$ \\ †dmitriev.sergey.v@gmail.com
}

${ }^{1}$ Institute for Metals Superplasticity Problems RAS, 39 S. Khalturin St., Ufa, 450001, Russia

${ }^{2}$ Bashkir State University, 32 Zaki Validi St., Ufa, 450076, Russia

${ }^{3}$ Mirny Polytechnic Institute (branch) of the North-Eastern Federal University, 5/1 Tikhonova St., Mirny, 678174, Russia

${ }^{4}$ National Research Tomsk State University, 36 Lenin Av., Tomsk, 634050, Russia

An interstitial atom placed into a close-packed atomic row is called crowdion. Crowdions are very efficient in mass transfer in the crystal lattice since they are highly mobile, soliton-like objects. It has been demonstrated recently that single interstitial atom can move along a close-packed atomic row with a supersonic speed in two different modes, either as a classical 1-crowdion or as a 2 -crowdion. The difference is that in the latter case two atoms move with a high speed at the same time, while in the former case only one atom has high speed. It has been shown that the 2-crowdion requires lesser energy to initiate mass transfer and it travels longer distance if it has same energy with the 1-crowdion. It is important to compare the efficiency of mass transfer by 2 -crowdions in different materials. Materials have different properties because the interatomic interactions between various atoms are different. In the present study we demonstrate that the most important characteristic of the interatomic potentials, that has effect on the crowdion path length, is the energy of the interatomic bond at the distance between two atoms equal to a half of the equilibrium interatomic distance. This conclusion is justified by the condition of self-focusing propagation of supersonic crowdions, that is the collision velocity of the atoms should not exceed the value when they approach each other closer than half interatomic distance. As an example, mass transfer by 1- and 2-crowdions is considered in two-dimensional triangular lattice with Morse and Born-Mayer potentials.

Keywords: interstitial, crowdion, mass transfer, molecular dynamics.

\section{Introduction}

Many physical processes in crystals, e.g., plastic deformation [1-7], structure transformation during heat treatment [8] and swelling under irradiation [9-13] are accompanied by the transport of mass over crystal lattice. Vacancy diffusion contributes noticeably to the mass transfer in the crystals at elevated temperatures $[8,14,15]$. Concentration of interstitial atoms in thermal equilibrium is considerably smaller than that of vacancies since the former have higher energy, but the role of interstitials considerably increases in far-from-equilibrium processes. Migration energy of interstitials can be relatively high [16] or very low when they are located in close packed atomic rows [17], in the latter case they are called crowdions. Interestingly, crowdions usually have lower potential energy than immobile interstitials $[17,18]$. Crowdions can be at rest, but they also can move at subsonic or supersonic speeds [19-21].

Stationary or subsonic crowdions have a kink profile, whose width is from half a dozen to a dozen atoms. Classical supersonic crowdions are strongly localized on single atom $[21,22]$. Crowdions play a very important role in relaxation processes associated with mass and energy transfer [6, 23-30]. It has been shown that clusters of interstitials formed during irradiation have very high mobility [23-30].

Moving excitations in crystals have recently attracted attention of researchers due to the experimentally observed effect of annealing of defects deep inside a germanium single crystal during surface plasma treatment [31], as well as due to exploration of the nature of tracks of particles that can be seen in mica crystals [21,32-36]. In particular, discrete breathers [37-43] and crowdions [21] as moving excitations in mica were discussed. The dynamics and collisions of supersonic crowdions in a two-dimensional lattice were analyzed in [44-49]. It was shown that moving discrete breathers and crowdions can transport electrons [50]. Crowdions can make a significant contribution to the mass transfer observed during severe plastic deformation (SPD) of metals and alloys. As it turned out, SPD, even at room temperature, can stimulate phase transformations occurring in the absence of deformation at much higher temperatures [51-54]. These phase transitions occur very quickly and are accompanied by accelerated mass transfer, which cannot be explained only by conventional mechanisms such as bulk and grain-boundary diffusion, even in the presence of an increased concentration of vacancies.

In the present study we continue the analysis of supersonic crowdion dynamics [45-49] by analyzing the effect of interatomic potentials on their path length. $2 \mathrm{D}$ triangular lattice is considered to speed up the simulations and taking into account that the results obtained earlier for 2D [47] and $3 \mathrm{D}$ [48] Morse lattices are in a very good qualitative agreement and differ only quantitatively. Besides, crowdions in $2 \mathrm{D}$ triangular lattices have been considered in relation to mica muscovite crystal, where nonlinear excitations can propagate in the potassium monatomic layer [37,39]. 


\section{Simulation setup}

A triangular two-dimensional lattice with interatomic distance $d$ is considered. As shown in Fig. 1, the $x$ axis is directed along close-packed direction.

Two pairwise interatomic potentials are considered.

The Morse potential is

$$
U_{\text {MR }}(r)=D\left(e^{-2 \alpha\left(r-r_{m}\right)}-2 e^{-\alpha\left(r-r_{m}\right)}\right) .
$$

Here $U_{\text {MR }}$ is the potential energy of two interacting atoms, $r$ is the distance between them, and $\alpha, D$, and $r_{m}$ are the potential parameters. $U_{\mathrm{MR}}(r)$ has minimum at $r=r_{m}, D$ is the depth of the potential (binding energy), and $a$ defines the rigidity of the interatomic bond.

Similarly, the Born-Mayer potential is

$$
U_{\mathrm{BM}}(r)=B\left(e^{-\left(r-r_{c}-1\right) / \beta}-2 /\left(r-r_{c}\right)^{6}\right) .
$$

Here parameters are denoted as $\beta, B$, and $r_{c}$. The first one defines the bond rigidity, the second one the potential depth, and the third one the equilibrium interatomic distance. Parameters of the two potentials were chosen in a way to achieve

$$
\begin{gathered}
U_{\mathrm{MR}}\left(r_{\min }\right)=U_{\mathrm{BM}}\left(r_{\min }\right)=-1, \\
\frac{d U_{\mathrm{MR}}}{d r}\left(r_{\min }\right)=\frac{d U_{\mathrm{BM}}}{d r}\left(r_{\min }\right)=0, \\
\frac{d^{2} U_{\mathrm{MR}}}{d r^{2}}\left(r_{\min }\right)=\frac{d^{2} U_{\mathrm{BM}}}{d r^{2}}\left(r_{\min }\right),
\end{gathered}
$$

where without loss of generality we set $r_{\min }=1$. From Eqs. (3) - (5) it follows that both potentials have minimum at $r_{\min }=1$, and at the point of minimum both potentials have equal value of -1 and equal value of the second derivative. Physically this means that the equilibrium interatomic distance for both potentials is equal to 1 , the binding energy of both potentials is equal to 1 , and they both have same rigidity for small displicaments from equilibrium distance. These conditions are achieved for $D=r_{m}=1, \alpha=6$ for the Morse potential and $B=0.98091338226, \beta=0.0716924$, and $r_{c}=-0.02142746$ for the Born-Mayer potential. The mass of an atom in both crystals is $M=1$, which can always be achieved by a proper choice of the time unit. The cut-off radius is $5 r_{m}$. Then the equilibrium interatomic distance

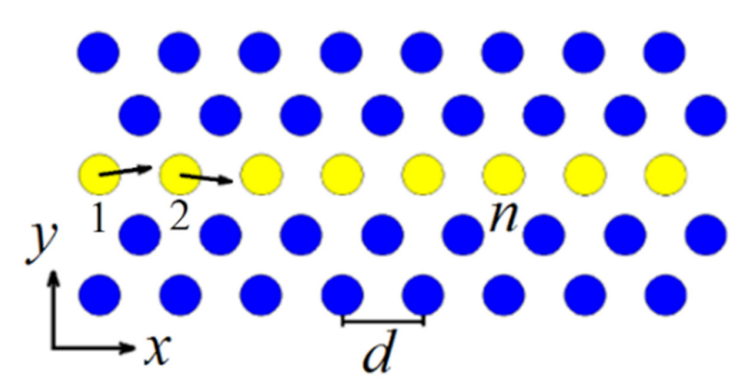

Fig. 1. Atoms of a triangular lattice on the $x y$ plane. The $x$ axis is directed along a close-packed atomic row, $d$ is the interatomic distance or diameter of an atom. Atoms in one close-packed row (shown lighter) are numbered by the index $n$. The initial conditions for initiating a 2-crowdion are shown: atoms 1 and 2 have initial velocities with components $\left(V_{0}, \varepsilon V_{0}\right)$ and $\left(V_{0},-\varepsilon V_{0}\right)$, where $\varepsilon \ll 1$. in the Morse lattice is $d=0.9955675$ and in the BornMayer lattice is $d=0.9908151$. The Morse and Born-Mayer potentials are shown in Fig. 2 by the solid and dashed lines, respectively. Inset shows the repulsive part of the potentials. It can be seen that the attractive parts of the potentials $(r>1)$ are close to each other, but the repulsive parts $(r<1)$ deviate noticeably. In the study of crowdions the repulsive part of the potential is important, since the interstitial atom produces local compression.

The equations of motion of atoms were integrated using the Stormer method of the sixth order of accuracy with a time step equal to $\tau=10^{-3}$. No thermal vibrations were introduced, that is, the simulations were performed at a temperature of $0 \mathrm{~K}$. Periodic boundary conditions are used.

1-crowdion was initiated by giving initial velocity to a single atom $\left(V_{0}, \varepsilon V_{0}\right)$, where $V_{0}$ is the initial velocity along the close-packed atomic row oriented along the $x$-axis and $\varepsilon \ll 1$, so that a small component of the velocity vector in the lateral direction is added in order to analyze the crowdion selffocusing. The initial conditions for initiating a 2-crowdion are shown in Fig. 1: atoms 1 and 2 have initial velocities with components $\left(V_{0}, \varepsilon V_{0}\right)$ and $\left(V_{0},-\varepsilon V_{0}\right)$, where $\varepsilon \ll 1$. The value $\varepsilon=10^{-6}$ was adopted. The energy given to the system by the excitation of crowdions is equal to the kinetic energy of the excited atoms,

$$
E_{0}=N M V_{0}^{2} / 2
$$

where $N=1$ and 2 for 1 - and 2 -crowdions, respectively. Recall that the mass of an atom is $M=1$ in our simulations.

\section{Condition of self-focusing atomic collisions}

When a supersonic crowdion moves along a close-packed atomic row, the atoms collide at a high speed. Their collisions can be self-focusing or defocusing.

The problem of self-focusing collisions in a chain of hard balls was solved in [55]. The balls of diameter $d$ are arranged in a row at a distance $s$ from each other. Suppose that the first ball is launched not strictly along the chain, but at a certain angle $\theta$. Exact geometrical calculations show that if $d>s$, then the sequence of collisions will be self-focusing, that is, the direction of the velocity vector of the next balls will exponentially quickly approach the axis of the chain. If this condition is violated, the collisions will be defocusing,

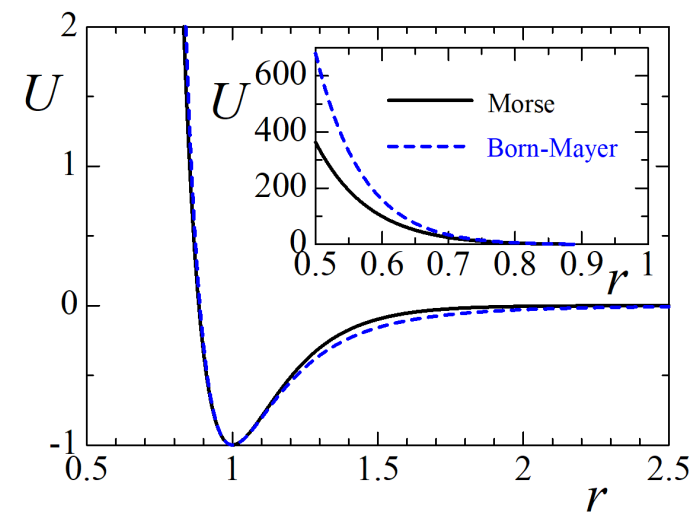

Fig. 2. (Color online) Morse and Born-Mayer potentials shown by the solid and dashed lines, respectively. Inset shows the repulsive part of the potentials. Potential parameters are chosen in a way to satisfy Eqs. (3) - (5) for $r_{\min }=1$. 
that is, an arbitrarily small initial deviation of the velocity vector from the axis of the chain will increase exponentially with subsequent collisions.

For a crystal in equilibrium, in a close-packed row the atoms of diameter $d$ have interatomic distance $d$, while the distance $s$ between them is zero (see Fig. 3, the upper panel). The atoms are not hard balls and their effective diameter depends on the collision velocity, and it is smaller for higher collision velocity. Taking into account the criterion of selffocusing collisions of hard balls, the criterion of self-focusing atomic collisions can be formulated as follows: the speed of collision should not exceed the value at which centers of atoms come closer than half of their diameter (see Fig. 3, the lower panel):

$$
d_{\min }>\frac{d}{2} .
$$

This self-focusing condition for atoms, in contrast to the condition for hard balls, is not strict, but it works quite well at least for the Morse pairwise interactions as shown in [48].

Having in mind the self-focusing condition (7), we now discuss the effect of the interatomic potentials on the crowdion dynamics. The inset in Fig. 2 shows that the repulsive parts of the Morse and Born-Mayer potentials are noticeably different. Since the atoms in the self-focusing collisions can approach each other, as maximum, by half atomic diameter (in our case $d=1)$, we note that at $U_{\mathrm{MR}}(d / 2)=363$, while $U_{\mathrm{BM}}(d / 2)=680$, which is almost two times greater. This means that the atoms in the Born-Mayer lattice can have almost two times greater energy not violating the self-focusing condition. Having this in mind one can expect that the Born-Mayer lattice can support self-focusing crowdions with higher energy and thus, propagating longer distances. In the following Section this conjecture will be supported by the numerical data.

\section{Results and discussion}

Crowdion motion is a periodic collision of atoms. If the frequency of atomic collisions is above the phonon spectrum, the radiation of phonons by the moving crowdion will be minimal and the energy loss will be mainly due to formation of the shock waves. That is why it is important to

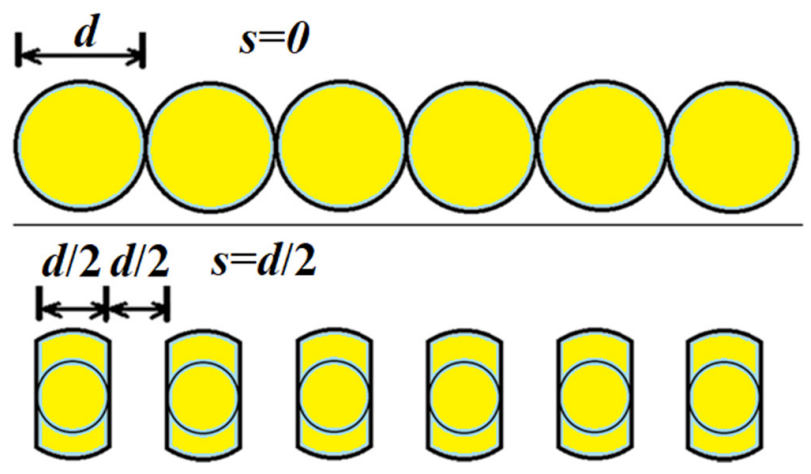

Fig. 3. To the discussion of self-focusing atomic collisions in a closepacked chain of atoms. The atoms have diameter $d$ and there is no space between them $(s=0)$. The effective diameter of atoms depends on the speed of their collision. The criterion for self-focusing collisions can be formulated as follows: the speed of their collisions should not exceed the value at which the centers of the atoms are closer than half of their diameter $(s=d / 2)$. compare the highest phonon frequency for the Morse and Born-Mayer lattices. The density of phonon states (DOS) for the two considered lattices is presented in Fig. 4. It can be seen that the highest phonon frequencies of the lattices are close: 3.43 for the Morse lattice, and 3.60 for the BornMayer lattice. This means that the conditions of radiationfree propagation for both lattices are close.

The main result of this study is presented in Fig. 5, where the crowdion path length measured in the interatomic distances, $N$, is shown as the function of (a) initial velocity $V_{0}$ of excited atoms and (b) initial energy $E_{0}$ given to the system. Results for the Born-Mayer (Morse) potential are presented by squares (circles). Empty symbols are for 1-crowdion and filled ones for 2-crowdion. For brevity we use the notations MR-1 and MR-2 for the 1- and 2-crowdions in the Morse lattice and, similarly, BM-1 and BM-2 for the 1and 2-crowdions in the Born-Mayer lattice. The dependence

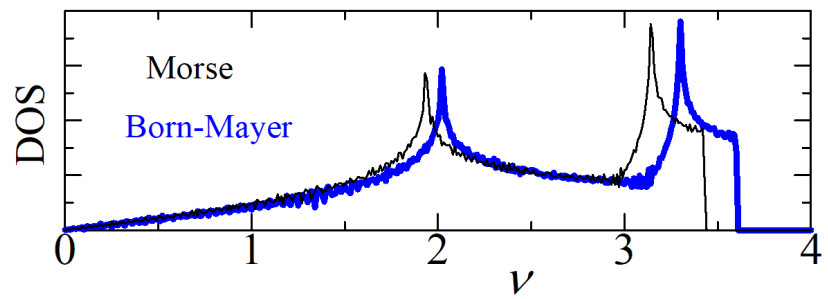

Fig. 4. (Color online) Density of phonon states for the twodimensional triangular lattice: thin black curve for the Morse potential, thick blue curve for the Born-Mayer potential.
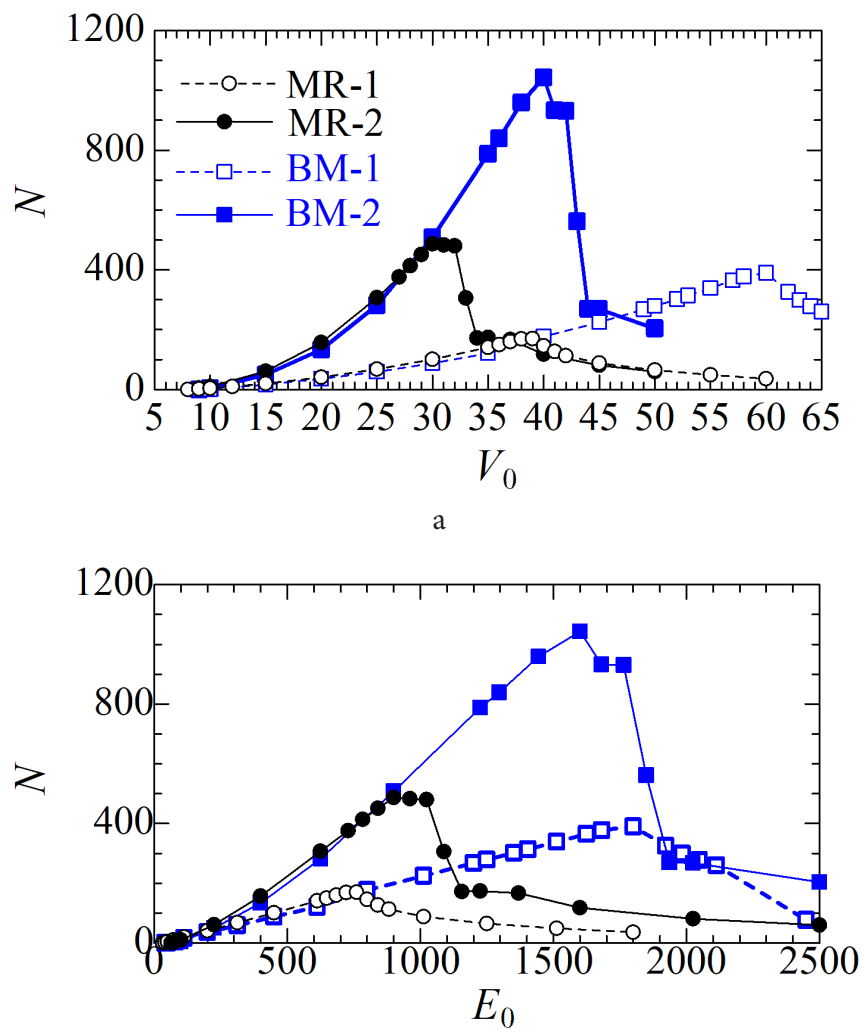

b

Fig. 5. (Color online) Crowdion path length in interatomic distances as the function of initial velocity of the excited atoms $V_{0}$ (a) and initial energy of the excited atoms $E_{0}(b)$. Open and filled circles are for the 1- and 2-crowdions, respectively, moving in the Morse crystal. Open and filled squares are for the 1- and 2-crowdions, respectively, moving in the Born-Mayer crystal. 
$N\left(E_{0}\right)$ is almost linear for the energies $E_{0}<E^{\star}$, where $E^{\star}=750$ for MR-1, $E^{\star}=900$ for MR-2, $E^{\star}=1800$ for BM-1, and $E^{\star}=1600$ for BM-2. For $E_{0}>E^{\star}$ the path length of the crowdions reduces considerably. This is because for $E_{0}>E^{\star}$ the condition of selffocusing propagation (7) is violated. It is clearly seen that in all cases 2 -crowdions have 2 to 2.5 times greater path length than 1-crowdions launched with the same initial energy $E_{0}$. Interestingly, for the initial velocity $V_{0}<39$ for 1 -crowdion and for $V_{0}<30$ for 2-crowdion the results obtained with the two different interatomic potentials are very close. However, for larger initial velocities both 1 - and 2-crowdions travel longer distances in the Born-Mayer lattice. This happens because for the Born-Mayer potential, as it was pointed out in Sec. 2, the energy $U_{\mathrm{BM}}(0.5)=680$ is considerably greater than $U_{\mathrm{MR}}(0.5)=363$, see the inset in Fig. 2 . This implies that in the Born-Mayer lattice self-focusing condition is satisfied for higher energy collisions. Having higher initial energies of self-focusing propagation, crowdions travel longer distances in the Born-Mayer latice.

\section{Conclusions}

Molecular dynamics simulations undertaken in this study demonstrate that actual profile of the repulsive part of the interatomic potential has little effect on the path length of 1or 2-crowdions, but the value of the potential at the distance equal to half interatomic distance strongly affects the crowdion path length. This is the main result of the present study. The explanation of this result is simple: self-focusing collisions in the Born-Mayer lattice take place for higher collision velocities and thus, for higher energies of crowdions. Crowdions with higher energy travel longer distances (see Fig. $5 \mathrm{~b}$ ) in the regime of self-focusing propagation.

This study also confirms that the 2-crowdions have 2 to 2.5 times greater path length then the 1-crowdions with the same initial energy. This phenomenon has been explained in $[45-49,56]$ through the fact that 2-crowdion with the same energy features smaller collision velocities between atoms and this is favorable for the self-focusing propagation of 2-crowdions.

As a continuation of this study, the criterion of the efficiency of mass transfer by crowdions deduced here for the two-dimensional lattices with pairwise potentials can be verified for various metals with many-body interactions [57].

Acknowledgements. E.A. Korznikova thanks the Grant of the President of the Russian Federation for state support of young Russian scientists, No. MD-3639.2019.2, for financial support (discussion of the results, writing the article). The work of A.S. Semenov (calculations, discussion of the results) was carried out under financial support of the Russian Science Foundation, grant No. 18-72-00006. This work was partially supported by the State assignment of IMSP RAS.

\section{References}

1. V.L. Indenbom, V. M. Chernov. Phys. Status Solidi (a). 14, 347 (1972). Crossref

2. V.V. Pokropivny, V.V. Skorokhod, A. V. Pokropivny. Model. Simul. Mater. Sc. 5, 579 (1997). Crossref
3. V.D. Natsik, S. N. Smirnov. Low Temp. Phys. 42, 207 (2016). Crossref

4. M. Kiritani. J. Nucl. Mater. 276, 41 (2000). Crossref

5. I. Salehinia, D. F. Bahr. Scripta Mater. 66, 339 (2012). Crossref

6. V.G. Kononenko, V.V. Bogdanov, A.N. Turenko, M. A. Volosyuk, A. V. Volosyuk. Probl. At. Sci. Tech. 104, 15 (2016).

7. A. Korbel, W. Bochniak. Int. J. Mech. Sci. 128, 269 (2017). Crossref https://doi.org/10.1016/j.ijmecsci.2017.04.006

8. H. Mehrer. Diffusion in Solids. Springer-Verlag, Berlin (2007) 651 p. Crossref

9. A.E. Sand, S. L. Dudarev, K. Nordlund. Europhys. Lett. 103, 46003 (2013). Crossref

10. X. Yi, M. L. Jenkins, K. Hattar, P. D. Edmondson, S. G. Roberts. Acta Mater. 92, 163 (2015). Crossref

11. Z. Zhang, K. Yabuuchi, A. Kimura, J. Nucl. Mater. 480, 207 (2016). Crossref

12. T. Koyanagi, N. A. P. K. Kumar, T. Hwang, L. M. Garrison, X. Hu, L. L. Snead, Y. Katoh. J. Nucl. Mater. 490, 66 (2017). Crossref

13. A. Xu, D.E. J. Armstrong, C. Beck, M.P. Moody, G. D. W. Smith, P.A. J. Bagot, S. G. Roberts. Acta Mater. 124, 71 (2017). Crossref

14. E. Korznikova, E. Schafler, G. Steiner, M. J. Zehetbauer. TMS Annual Meeting 2006. San Antonio, Texas (2006) p. 97.

15. B. Liu, L. Bai, E.A. Korznikova, S. V. Dmitriev, A. W.-K. Law, K. Zhou. J. Phys. Chem. C. 121, 13876 (2017). Crossref

16. D. A. Terentyev, T. P. C. Klaver, P. Olsson, M.-C. Marinica, F. Willaime, C. Domain, L. Malerba. Phys. Rev. Lett. 100, 145503 (2008). Crossref

17. H. R. Paneth. Phys. Rev. 80, 708 (1950). Crossref

18. P. M. Derlet, D. Nguyen-Manh, S. L. Dudarev. Phys. Rev. B. 76, 054107 (2007). Crossref

19. A. M. Kosevich, A.S. Kovalev. Solid State Commun. 12, 763 (1973). Crossref

20. A.S. Davydov, A. V. Zolotariuk. Phys. Scripta. 30, 426 (1984). Crossref

21. J. F. R. Archilla, Y. A. Kosevich, N. Jimenez, V. J. SanchezMorcillo, L.M. Garcia-Raffi. Phys. Rev. E. 91, 022912 (2015). Crossref

22. Yu. A. Kosevich, R. Khomeriki, S. Ruffo. Europhys. Lett. 66, 21 (2004). Crossref

23. Y.N. Osetsky, D. J. Bacon, A. Serra. Phil. Mag. Lett. 79, 273 (1999). Crossref

24. S. Han, L.A. Zepeda-Ruiz, G. J. Ackland, R. Car, D. J. Srolovitz. Phys. Rev. B. 66, 220101 (2002). Crossref

25. H. Abe, N. Sekimura, Y. Yang. J. Nucl. Mater. 323, 220 (2003). Crossref

26. S. L. Dudarev. Philos. Mag. 83, 3577 (2003). Crossref

27. Y.N. Osetsky, D. J. Bacon, A. Serra, B.N. Singh, S. I. Golubov. Philos. Mag. 83, 61 (2003). Crossref

28. D. A. Terentyev, L. Malerba, M. Hou. Phys. Rev. B. 75, 104108 (2007). Crossref

29. W.H. Zhou, C.G. Zhang, Y.G. Li, Z. Zeng. Scientific Reports. 4, 5096 (2014). Crossref

30. W. H. Zhou, C. G. Zhang, Y. G. Li, Z. Zeng. J. Nucl. Mater. 453, 202 (2014). Crossref 
31. J. F. R. Archilla, S. M. M. Coelho, F. D. Auret, V. I. Dubinko, V. Hizhnyakov. Physica D. 297, 56 (2015). Crossref

32. F. M. Russell. Nature (London). 217, 51 (1967). Crossref

33. F. M. Russell. Phys. Lett. A. 130, 489 (1988). Crossref

34. F. Russell. Nucl. Tracks. Rad. Meas. 15, 41 (1988). Crossref

35. D. Schlößer, K. Kroneberger, M. Schosnig, F. M. Russell, K. O. Groeneveld. Radiat. Meas. 23, 209 (1994). Crossref

36. F. M. Russell, J.C. Eilbeck. Europhys. Lett. 78, 10004 (2007). Crossref

37. J. Bajars, J. C. Eilbeck, B. Leimkuhler. Physica D. 301-302, 8 (2015). Crossref

38. J. Bajars, J.C. Eilbeck, B. Leimkuhler. Springer Series Mate. 221, 35 (2015). Crossref

39. J. L. Marin, F. M. Russell, J. C. Eilbeck. Phys. Lett. A. 281, 21 (2001). Crossref

40. S.V. Dmitriev, E.A. Korznikova, J.A. Baimova, M. G. Velarde. Phys. Usp. 59, 446 (2016). Crossref

41. R. T. Murzaev, R. I. Babicheva, K. Zhou, E. A. Korznikova, S. Y. Fomin, V.I. Dubinko, S. V. Dmitriev. Eur. Phys. J. B. 89, 168 (2016). $\underline{\text { Crossref }}$

42. E. Barani, I. P. Lobzenko, E. A. Korznikova, E. G. Soboleva, S. V. Dmitriev, K. Zhou, A. M. Marjaneh. Eur. Phys. J. B. 90, 38 (2017). Crossref

43. P.V. Zakharov, M.D. Starostenkov, A.M. Eremin, E. A. Korznikova, S. V. Dmitriev. Phys. Solid State. 59, 223 (2017). Crossref

44. A. P. Chetverikov, W. Ebeling, M.G. Velarde. Physica D. 240, 1954 (2011). Crossref

45. A. M. Bayazitov, E.A. Korznikova, I.A. Shepelev, A.P. Chetverikov, K.S. Khadiullin, E.A. Sharapov, P.V. Zakharov, S. V. Dmitriev. IOP Conference Series: Materials Science and Engineering. 447, 012040 (2018). Crossref
46. A. Moradi Marjaneh, D. Saadatmand, I. Evazzade, R. I. Babicheva, E. G. Soboleva, N. Srikanth, K. Zhou, E. A. Korznikova, S. V. Dmitriev. Phys. Rev. E. 98, 023003 (2018). Crossref

47. S. V. Dmitriev, E. A. Korznikova, A.P. Chetverikov, J.Exp. Theor. Phys. 126, 347 (2018). Crossref

48. S. V. Dmitriev, N.N. Medvedev, A.P. Chetverikov, K. Zhou, M.G. Velarde. Phys. Status Solidi RRL. 11, 1700298 (2017). Crossref

49. A.P. Chetverikov, I.A. Shepelev, E.A. Korznikova, A.A. Kistanov, S.V. Dmitriev, M.G. Velarde. Computational Condensed Matter. 13, 59 (2017). Crossref

50. Yu. A. Kosevich. IOP Conf. Series: Journal of Physics: Conf. Series. 833, 012021 (2017). $\underline{\text { Crossref }}$

51. B. B. Straumal, X. Sauvage, B. Baretzky, A. A. Mazilkin, R. Z. Valiev. Scripta Mater. 70, 59 (2014). Crossref

52. B. Straumal, A. Korneva, P. Zieba. Arch. Civil Mech. Eng. 14, 242 (2014). Crossref

53. B. B. Straumal, A.R. Kilmametov, Y.O. Kucheev, K. I. Kolesnikova, A. Korneva, P. Zieba, B. Baretzky. Jetp Lett. 100, 376 (2014). $\underline{\text { Crossref }}$

54. C.M. Cepeda-Jimenez, J.I. Beltran, A. Hernando, M. A. Garcia, F. Yndurain, A. Zhilyaev, M. T. Perez-Prado. Acta Mater. 123, 206 (2017). Crossref

55. R. I. Garber, A. I. Fedorenko. Sov. Phys. Usp. 7, 479 (1965). Crossref

56. E. A. Korznikova, I. A. Shepelev, A.P. Chetverikov, S. V. Dmitriev, S. Y. Fomin, K. Zhou. J. Exp. Theor. Phys. 127, 1009 (2018). Crossref

57. A.M. Iskandarov, N.N. Medvedev, P.V. Zakharov, S. V. Dmitriev. Comput. Mater. Sci. 47, 429 (2009). Crossref 